05;15

\title{
Нейросетевой метод прогнозирования образования дефектов на поверхности тонких пленок ITO при механических \\ нагрузках
}

\author{
(ㄱ Д.А. Кириенко, О.Я. Березина
}

Петрозаводский государственный университет, Петрозаводск, Россия

E-mail: kirienko@petrsu.ru

Поступило в Редакцию 11 января 2018 г.

Предлагается метод определения числа дефектов, возникающих под воздействием сжимающих и растягивающих напряжений при изгибе тонких прозрачных проводящих покрытий на полимерных подложках. Алгоритм определения основан на использовании математических методов искусственных нейронных сетей. Сеть натренирована на расчет средней плотности дефектов на единицу длины при входных параметрах, соответствующих размерам пленки и подложки, поверхностному сопротивлению проводящего покрытия и радиусу изгиба. Применение метода позволяет определить среднюю плотность дефектов с высокой точностью.

DOI: 10.21883/PJTF.2018.09.46069.17207

Тонкие пленки оксида индия с примесью олова (indium tin oxide ITO) благодаря прозрачности в видимой области спектра и низкому удельному сопротивлению находят применение при изготовлении светоизлучающих полупроводниковых элементов и оптоэлектронных приборов. В последнее время большое внимание уделяется исследованию методов получения прозрачных проводящих покрытий на полимерных подложках, которые могут использоваться как электроды при изготовлении устройств гибкой оптоэлектроники. При изготовлении электродов на основе тонких пленок ITO важны не только их электрические и оптические свойства, но и устойчивость к внешним изгибающим или растягивающим нагрузкам, так как это напрямую влияет на долговечность и надежность изготавливаемых устройств. Изучению влияния деформации на параметры ITO посвящено мно- 
жество исследований [1-6], что указывает на актуальность данной проблемы.

Полимерные подложки из полиэфирсульфона, полиэтилентерефталата (PET) и полиэтиленнафталата, которые чаще всего используются в устройствах гибкой электроники, обладают большей эластичностью по сравнению с ITO. Поэтому в процессе нагружения подложка обычно испытывает только упругую деформацию, а пленка деформируется пластически. При этом на пленке возможно образование складок (гофрирование) и трещин, приводящих к уменьшению ее прозрачности и проводимости [2-9]. Механизмы деформации и разрушения тонкопленочных структур хорошо изучены, но ввиду того, что сложно учесть все процессы, происходящие в материале, изучение усталостного поведения тонких пленок проводится экспериментально. Целью настоящей работы является демонстрация принципиальной возможности использования математического метода искусственных нейронных сетей (ИНС) для определения числа дефектов, возникающих под воздействием сжимающих и растягивающих напряжений при изгибе пленки.

В работе использованы две ИНС $\left(A N_{1}\right.$ и $\left.A N_{2}\right)$, реализованные на базе сети с одним скрытым слоем с сигмоидальной функцией активации. $A N_{1}$ моделирует образование дефектов при сжатии пленки, $A N_{2}$ - при растяжении. Каждая нейронная сеть состоит из шести входных нейронов, восемнадцати скрытых и одного нейрона на выходном слое. Каждый входной нейрон имеет связь со всеми скрытыми, которые в свою очередь соединены с выходным. Число скрытых нейронов определялось эмпирическим путем с учетом роста времени вычислений. Весовые коэффициенты нейронов определялись методом обратного распространения, использующим алгоритм градиентного спуска. В модели предполагается, что исследуемый образец, представляющий собой прямоугольную полимерную РЕТ-подложку, на которую нанесено ITO-покрытие, жестко закреплен с двух сторон. Изгиб образца осуществляется путем сведения концов друг к другу. Также при условии малой толщины пленок предполагается, что ITO испытывает сжатие, когда центр изгиба располагается со стороны пленки, и растяжение, когда центр изгиба находится со стороны подложки. Таким образом, входные нейроны отождествляются с длиной $(L)$ и шириной $(W)$ образца, толщиной подложки $\left(h_{s}\right)$ и пленки ITO $\left(h_{f}\right)$, смещением центральной точки образца при изгибе относительно

Письма в ЖТФ, 2018, том 44, вып. 9 
недеформированного состояния $(D)$ (стрела прогиба), поверхностным сопротивлением ITO $\left(r_{s}\right)$. Последний параметр добавлен к входным данным, поскольку подвижность носителей заряда связана с напряжением дислокаций и внутренними дефектами в ITO, возникшими в пленке при ее нанесении на подложку [8]. Модель ограничена одним способом формирования слоя ITO на подложке, поэтому технологические параметры изготовления образца не учитываются. Выходной нейрон каждой сети отождествлен со средним числом дефектов на единицу длины пленки $\left(C_{d}\right)$. Дефекты для $A N_{1}$ определялись как складки при воздействии сжимающих напряжений, а для $A N_{2}$ - как трещины при растяжении.

Тренировка нейронных сетей производилась в два этапа: 1) первоначальное обучение и подбор весовых коэффициентов нейронов по результатам моделирования напряжений, возникающих в пленке при изгибе; 2) адаптация ИНС на основании экспериментальных данных.

Моделирование напряжений, возникающих в пленке при изгибе, проведено при помощи программы ANSYS Workbench с использованием метода конечных элементов [2,3,6,7]. Суть метода заключается в разбиении объема исследуемого образца на гексагональные элементы малого размера. Значения напряжений, возникающих в каждом элементе, рассчитываются на основании механических характеристик и размеров системы пленка-подложка, а также смещения относительно недеформированного положения. При деформациях сжатия и растяжения дефекты на поверхности пленки имеют распределение с периодом $\lambda[9-11]$

$$
\lambda=2 \pi h_{f}\left(\frac{\bar{E}_{f}}{3 \bar{E}_{s}}\right)^{1 / 3},
$$

где $h_{f}$ - толщина пленки, $\bar{E}_{f}=E_{f} /\left(1-v_{f}^{2}\right)$ и $\bar{E}_{s}=E_{s} /\left(1-v_{s}^{2}\right)-$ модули плоской деформации пленки и подложки, $E_{f}=116 \mathrm{GPa}$ и $v_{f}=0.35, E_{s}=3 \mathrm{GPa}$ и $v_{s}=0.47-$ модули упругости и коэффициенты Пуассона для пленки ITO и подложки PET $[1,6]$. Для численного моделирования напряжений дробление объема системы пленка-подложка осуществлялось так, чтобы размер одного элемента был сопоставим с $\lambda$. Условие образования дефектов в пленке принималось как превышение возникающих в результате изгиба напряжений $\sigma$ над критическими

$6^{*}$ Письма в ЖТФ, 2018, том 44, вып. 9 
значениями $\sigma_{c}$ для сжатия и $\sigma_{t}$ для растяжения [2,9-12],

$$
\sigma_{c}=\frac{\pi^{2}}{3}\left(\frac{h_{f}}{\lambda / 2}\right)^{2} \bar{E}_{f}, \quad \sigma_{t}=\sqrt{\frac{G \bar{E}_{f}}{1.258 \pi h_{f}}}
$$

где $G=35 \mathrm{~J} / \mathrm{m}^{2}[1]$ - удельная работа разрушения пленки ITO. Моделирование образования дефектов в пленке в зависимости от параметров системы пленка-подложка в программе ANSYS производилось при следующих диапазонах входных данных: $W=0.5-2 \mathrm{~cm}$ с шагом $0.25 \mathrm{~cm}, L=4-8 \mathrm{~cm}$ с шагом $0.25 \mathrm{~cm}, h_{f}=50-500 \mathrm{~nm} \mathrm{с} \mathrm{шагом} 10 \mathrm{~nm}$, $D=0-2 L / 3$ с шагом $0.1 \mathrm{~mm}, h_{s}=200-100 \mu \mathrm{m}$ с шагом $50 \mu \mathrm{m}$. По результатам моделирования было подсчитано число дефектов $(c)$ и определена средняя плотность дефектов на единицу длины образца $\left(C_{d}=c / L\right)$. Для обучения нейронной сети множество входных данных было дополнено значениями поверхностного сопротивления $r\left(h_{f}\right)$

$$
r\left(h_{f}\right)=-1.654 \cdot 10^{19} h_{f}^{3}-2.385 \cdot 10^{14} h_{f}^{2}-1.626 \cdot 10^{6} h_{f}-148.087 .
$$

Аппроксимация $r\left(h_{f}\right)$ была получена на основе экспериментально измеренных четырехзондовым методом поверхностных сопротивлений образцов ITO-пленок на РЕТ-подложке.

Для того чтобы ИНС выдавала результаты, наиболее близкие к реальным, после обучения был произведен процесс адаптации с использованием экспериментальных образцов. Пленки с различной толщиной ITO, полученные методом магнетронного распыления на холодную PETподложку, изгибались таким образом, чтобы пленка ITO была внутри (сжималась) или снаружи (растягивалась). Стрела прогиба $D$ изменялась от 0 до $50 \mathrm{~mm}$. Затем с помощью оптического микроскопа, обеспечивающего разрешение $2 \mu \mathrm{m}$, определялось число складок или трещин в различных областях поверхности пленки. Полученное значение усреднялось и использовалось для корректировки весовых коэффициентов нейронов. Для адаптации использовались группы образцов $(\alpha, \beta)$ со следующими характеристиками: $L_{\alpha}=4 \mathrm{~cm}, W_{\alpha}=1 \mathrm{~cm}, h_{f \alpha}=80 \mathrm{~nm}$, $h_{s \alpha}=250 \mu \mathrm{m}, r_{\alpha}=150 \Omega / \square$ и $L_{\beta}=8 \mathrm{~cm}, W_{\beta}=1 \mathrm{~cm}, h_{f \beta}=220 \mathrm{~nm}$, $h_{s \beta}=250 \mu \mathrm{m}, r_{\beta}=137 \Omega / \square$. Апробация работы нейронной сети производилась на третьей группе образцов $(\gamma)$ с характеристиками $L_{\gamma}=6 \mathrm{~cm}$, $W_{\gamma}=1.5 \mathrm{~cm}, h_{f \gamma}=110 \mathrm{~nm}, h_{s \gamma}=500 \mu \mathrm{m}, r_{\gamma}=143 \Omega / \square$.

Письма в ЖТФ, 2018, том 44, вып. 9 

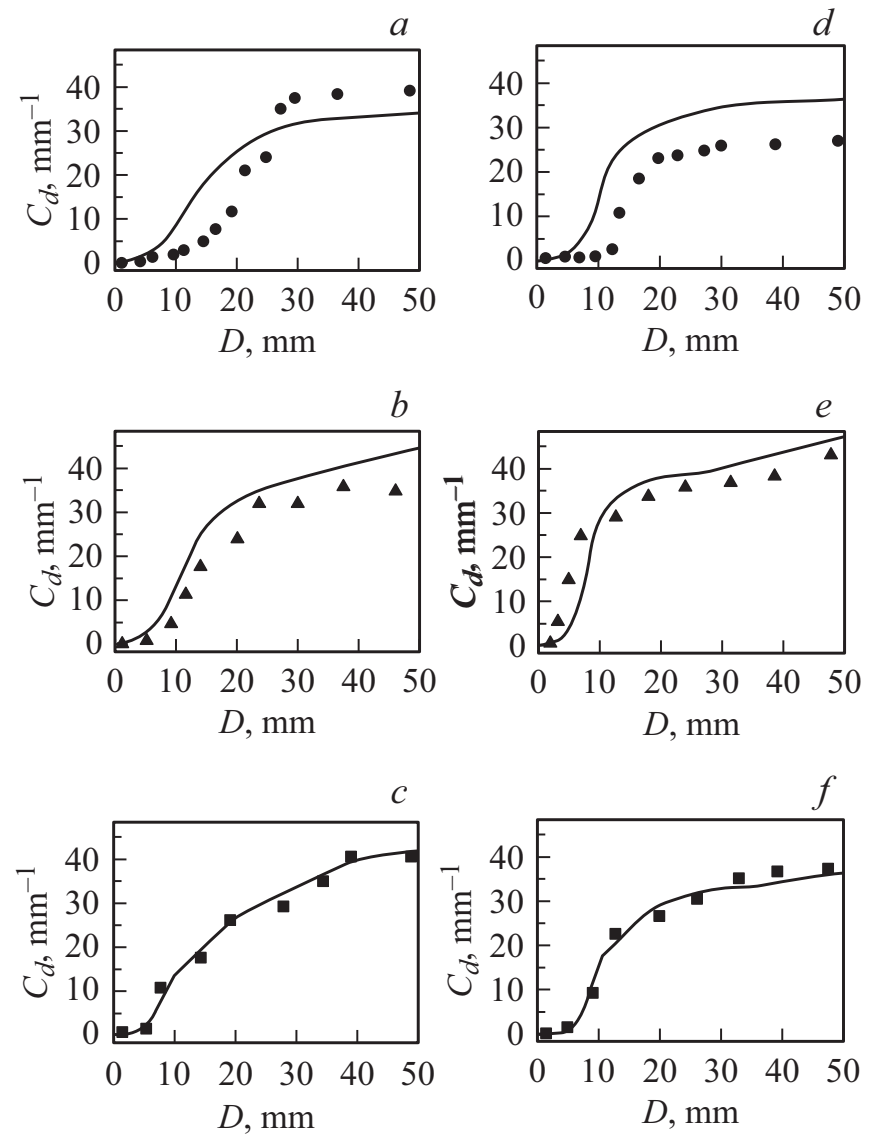

Зависимость числа дефектов на единицу длины $C_{d}$ от изгиба $D$ пленки ITO при деформации сжатия $(a-c)$ и растяжения $(d-f)$. Сплошными линиями показаны результаты расчета ИНС: $a, d-$ после обучения, $b, c, e, f-$ после адаптации. Символы - экспериментальные данные для различных групп образцов: $a, d-\alpha ; b, e-\beta ; c, f-\gamma$.

Результаты работы ИНС на стадиях обучения, адаптации и апробации для деформации изгиба и сжатия представлены на рисунке. После обучения сети по данным моделирования зависимость средней

Письма в ЖТФ, 2018, том 44, вып. 9 
плотности дефектов от изгиба образца существенно отклоняется от экспериментальных результатов (см. рисунок, $a, d$ ). Это обусловлено тем, что в процессе моделирования было опущено множество факторов, влияющих на образование дефектов в пленке, а также существует погрешность в механических характеристиках материалов. Но данный этап тренировки позволил существенно сократить число образцов и измерений, необходимых для адаптации модели. После корректировки весовых коэффициентов по экспериментальным данным образцов $\alpha$ расчет ИНС стал ближе к истинному (см. рисунок, $b, e)$. Удовлетворительный расчет $C_{d}(D)$ произведен нейронной сетью после второго подэтапа адаптации с использованием экспериментальных данных образцов $\beta$. Отклонение $C_{d}(D)$ от расчетного значения для образов $\gamma$ (см. рисунок, $c, f$ ) составило не более $5 \%$, что свидетельствует о правильном обучении ИНС.

Таким образом, в работе исследована возможность использования нейросетевого подхода для определения числа дефектов, возникающих под воздействием сжимающих и растягивающих напряжений при изгибе тонких пленок оксидов на полимерной подложке. Преимущество применения методов ИНС заключается в возможности выполнения расчетов при неизвестных закономерностях входных и выходных параметров. Также предполагается, что в дальнейшем будет проведена корректировка архитектуры ИНС для определения устойчивости электрических и оптических свойств прозрачных проводящих покрытий к деформации и циклическим нагрузкам.

Работа выполнена при поддержке Российского фонда фундаментальных исследований (проект № 16-32-60090).

\section{Список литературы}

[1] Tran D.-P., Lu H.-I., Lin C.-K. // Surf. Coat. Technol. 2016. V. 283. P. 298-310.

[2] Harris K.D., Elias A.L., Chung H.-J. // J. Mater Sci. 2016. V. 51. N 6. P. 27712805.

[3] Lee C.-C., Huang P.-C., Wang K.-S. // Materials. 2016. V. 9. N 10. P. 850.

[4] Yeh M.-K., Chang L.-Y., Cheng H.-C., Wang P.-H. // Proc. Eng. 2014. V. 79. P. 189-193.

[5] Lim C.-Y., Park J.-K., Kim Y.-H., Han J.-I. // J. Int. Council Electr. Eng. 2012. V. 2. N 3. P. 237-241.

Письма в ЖТФ, 2018, том 44, вып. 9 
[6] Peng C., Jia Z., Bianculi D., Li T., Lou J. // J. Appl. Phys. 2011. V. 109. P. 103530.

[7] Amiri A., Belhouari M., Bounoua N., Achour T., Bouiadjra B.B. // Mech. Res. Commun. 2013. V. 47. P. 1-5.

[8] Adurodija F., Izumi H., Ishihara T., Yoshioka H., Motoyama M. // J. Mater. Sci.: Mater. Electron. 2001. V. 12. N 1. P. 57-61.

[9] Tada H., Paris P., Irwin G. The stress analysis of cracks handbook. 2nd ed. St. Louis: Del Research Corp., 1985.

[10] Lei J., Wang Y.-S., Gross D. // Int. J. Solids Struct. 2007. V. 44. P. 996-1012.

[11] Huang R., Suo Z. // J. Appl. Phys. 2002. V. 91. N 3. P. 1135-1142.

[12] Шгуров А.P., Панин А.В. // Физ. мезомеханика. 2009. Т. 12. № 3. С. 23-32. 